\title{
Transparencia y Publicidad en el Arbitraje de Inversión
}

\author{
Ignacio Torterola*
}

\section{SUMARIO}

1. Algunas Consideraciones Introductorias. 2. Transparencia y Publicidad en el Arbitraje de Inversión. 2.1. La Obligación de Transparencia y Publicidad en los Actos de Gobierno. 2.2. La Naturaleza Pública del Arbitraje Inversor-Estado. 2.2.1 Antecedentes Históricos. 2.2.2. La Práctica de los Tribunales de Inversión. 2.2.3. Resolución de la Comisión de Libre Comercio del TLCAN. Metalclad, Loewen, Methanex UPS. 2.2.4. Aguas del Tunari c. Bolivia y AASA y APSF (Suez) c. República Argentina. 2.2.5. La reforma de las Reglas de Arbitraje del CIADI de 2006 BiWater Gauff. 2.2.6. El Artículo 34(5) de las Reglas de Arbitraje CNUDMI 2010. 2.3. Hacia la Conformación de un Cuerpo Jurisprudencial. 3. Conclusiones.

- Ignacio Torteroia es abogado argentino. Desde el año 2000 es Miembro del Cucrpo Diplomático Argentino. A partir del año 2003 ha trabajado desde el Minisırio de Relaciones Exıcriores y la Procuración del Tesoro de la Nación como abogado en los casos relacionados a la emergencia económica en Argentina, entre ellos los casos CMS (Anulación), Euron (Anulaciỏn), Sempra (Anulación), Jivendi, Suez. National Grid, British Gas, Afctalpar, Wintershall, TSA Spectrum y Cominemal contra República Argentina (entre otros). Desde el año 2006 es el Énlace entre la Procuración del Tesoro de la Nación y el ClADI en la ciudad de Washington, DC. Las opiniones expresadas en este comentario son a titulo personal del autor, y en modo alguno iniçntan reflejar la posición del gobicrno de la Renública Angentina o de sus Ministerios y/o agencias. Algunas de las consideraciones expresadas en este articulo aparecen también en otro articulo del autor publicado en idioma inglés para la primera publicación de la Revista "Investment Treaty News" (ITN). Otras consideraciones, sin embargo. son propias de este articulo. Asimismo, mientras que el alcance de la presente publicación, en el caso de ITN la versión allí publicada refleja especificamente la problemática de las transparencia y publicidad en las nuevas Reglas de Arbitraje UNCITRAL. (2010). 


\section{Algunas Consideraciones Introductorias}

Este trabajo de investigación y opinión analiza una temática que ha sido frecuentemente motivo de ásperas discusiones en esta práctica judicial: las obligaciones de transparencia y publicidad asumidas por algunos Estados en sus compromisos internacionales o en sus leyes internas vs. la obligación de confidencialidad como característica peculiar del arbitraje como medio de solución de controversias. En otros términos, esta dicotomía surge, en opinión de este autor, de la naturaleza jurídica del arbitraje de inversión: ¿el "arbitraje de inversión" es arbitraje, en el sentido que lo expresa el arbitraje comercial o es un sistema de solución de controversias de carácter público al que el Estado se sujeta para resolver un tipo especial de controversia. Finalmente, es posible que sea ambos?

Recientemente, en torno a la discusión planteada por la controvertida decisión dictada en el caso Aguas del Aconquija c. República Argentinal y, especialmente en la Opinión Disidente del Sr. Jan Hendrik Dalhuisen, ${ }^{2}$ un conocido comentarista señalaba que

"han sido las partes en tratados bilaterales de inversión las que han consistentemente elegido el arbitraje comercial internacional como el foro para la solución de las controversias que se producen en su aplicación. Si quisieran adoptar un método diferente, siempre son libres de hacerlo. $\mathrm{Si}$, por el contrario, usaran el arbitraje comercial internacional, lo deberían usar tal cual como lo han encontrado. Los Tribunales del CIADI no son "cortes internacionales" ni se debe esperar que quienes los conforman asuman el rol de jueces". 3

1. Aguas del Aconquija SA y Vivendi SA c. República Argentina. Caso ClADI ARB/97/3. Segunda Decisión de Anulación de fecla 30 de julio de 2010.

2. Opinión disidente de Jan Hendrik Dalhuisen en Caso ARB/97/3 Op. Cit. p. 71.

3. Todd Weiler, en opinión expresada en ocasión de la decisión en el Caso Aguas del Aconquija. Traducción del Autor. OGEMID,17 de agosto de 2010. "It has been the parties to invesintent treaties who have consistently chosen international commercial arbitration as the foram for dispute resolution under them. If they want to adopt a different mechanism for dispute settement, they are ahways free to do so. If. however, they are going to use international commencial arbitration, they must take it as they find it. ICSID Tribunals are not 'international courts, 'nor can we expect those who populate them to take on the role of judges". 
Otro comentarista, en la misma ocasión, inmediatamente señala:

"para ampliar el debate, puede ser la inmunidad de los árbitros bajo el sistema CIADI un elemento para interpretar que estos ejercen jurisdicción internacional (en el modo de la CIJ y a diferencia de los tribunales ad hoc ... cuál es el criterio para que se constituyan en una corte internacional, la creación bajo un tratado ...."),

indicando que en su opinión es que el CIADI constituye una verdadera corte internacional.

En definitiva, aquí se explora la práctica de los tribunales en la consideración de sus obligaciones de transparencia y publicidad y, posteriormente, se analiza también la conveniencia de dotar de mayor publicidad al sistema arbitral, especialmente cuando se trata de controversias que involucran una parte estatal. Finalmente, se ofrecen algunas breves consideraciones personales.

\section{Transparencia y Publicidad en el Arbitraje de Inversión}

\subsection{La Obligación de Transparencia y Publicidad de los Actos de Gobierno}

En la generalidad de los arbitrajes con "partes estatales", y sin dudas en el caso de los arbitrajes de inversión, se debaten cuestiones que afectan la "cosa pública". La mayoría de los Estados democráticos modernos tiene -como consecuencia de estar regidos por principios republicanos de gobierno- obliga-

4. Sćbastien Manciaux, en opinión expresada en ocasión de la decisión de anulación en el Caso Aguas del Aconquija. OGEMID, 17 de agosto de 2010. "In order to brouden the debate, could this immunity of ICSID arbitrators be the suitable criterion to assert that ICSID tribunals are international jurisdiction (like the ICJ, and unlike ad hoc tribunals for instance)? Or is it ontly a consequence? If it is so, what could be the adequate criterion in order to qualify a jurisdiction as an international one (creation by a Treat): application of international Law: etc)?". 
ciones de información y de acceso a la información por parte de sus ciudadanos. En los casos en que existe regulación legal específica sobre la materia, como es el caso en la República Argentina, la publicidad de ciertos actos de gobierno no es ya una decisión del gobernante sino, verdaderamente, una obligación que le impone la ley.

En la República Argentina existe normativa que claramente establece el principio de publicidad de los actos de gobierno y el derecho al acceso a la información pública. Estas normas están contenidas en el texto de la propia Constitución Nacional, a través de los artículos 1, 33, 41, 42 y concordantes del Capítulo Segundo -que establece nuevos Derechos y Garantías- y del artículo 75 inciso 22, que incorpora con jerarquía constitucional diversos tratados internacionales sobre derechos humanos.

Aunque aquí describo el estadio de las obligaciones de publicidad y acceso a la información en Argentina, como dije anteriormente, la misma se encuentra presente en la mayoría de los Estados modernos.

\subsection{La Naturaleza Pública del Arbitraje Inversor-Estado}

\subsubsection{Antecedentes Históricos}

Históricamente, la práctica de los tribunales que han entendido en cuestiones relacionadas a los Estados actuando con ius imperii ha sido siempre pública, aplicándose este concepto a los tribunales arbitrales del siglo XIX como al fecundo trabajo de la Corte Internacional de Justicia y su predecesora la Corte Permanente de Justicia Internacional.

Asimismo, la mayoría de las comisiones bilaterales que han resuelto controversias pecuniarias contra Estados soberanos en el ámbito de la protección diplomática -como la Comisión Mixta 
México-Estados Unidos- y posteriormente bajo otros mecanismos especialmente diseñados como la Comisión de Compensaciones de las Naciones Unidas 5 y, especialmente, los Tribunales de Reparaciones Irán-Estados Unidos ${ }^{6}$ hicieron públicos sus laudos, algunos de ellos sesionando en una época que en mucho precede a la preocupación actual por la transparencia de los actos de gobierno.

\subsubsection{La Práctica de los Tribunales de Inversión}

El Convenio del CIADI establece que el laudo sólo se hará público con el consentimiento de las partes (Artículo 48(5) del Convenio CIADI). Sin embargo, a partir de aproximadamente el año 2000, también como consecuencia del avenimiento del uso masivo del internet, las decisiones arbitrales comenzaron a estar disponibles públicamente en sitios de internet, inclusive sin el consentimiento de las Partes involucradas en la controversia y, muy probablemente, a través de filtraciones que permitían los propios abogados representantes de las Partes en la controversia.

Este fenómeno no alcanza solamente a las decisiones bajo el sistema CIADI, sino que también impacta a procedimientos bajo otras reglas, especialmente casos bajo las Reglas de la CNUDMI. Esto justifica que un comentarista pueda decir que cierta información como "la existencia de la controversia, las partes y sus representantes legales, la naturaleza de la disputa y la composición del tribunal arbitral" encuentren su camino hacia la información de dominio público. ${ }^{7}$

5. Andrea, Gatlini. Thi: UN Comptensation Commission: Old Rules, New Procedures on War Reparations. EJIL, Vol. I3 No.1, $161-168$ (2002).

6. Caron \& Caplan \& Pellonpaa. Op. Cit. p. 9.

7. Mistelis, Loukas. Confidentiality and TiIIRD Party Particitation: UPS v CANADA and Methianex Corf. v. USA. En Inttanational. Investment Law and Arbitration. Leading Cases from tie ICSID, NAFTA, Bilateral. Treaties and Customary International Law. Todd Weiler (editor), Cameron May, 2005.[traducción del autor] “... mientras que la privacidad de los procedimientos en arbitraje de inversión todavia se reconoce, información tal como la existencia de la controversia, las partes y sus representantes legales, la naturaleza de la disputa y la composición del tribunal arbitral encuentran su camino hacia la información de dominio público". 


\subsubsection{Resolución de la Comisión de Libre Comercio del TLCAN. Metalclad, Loewen, Methanex y UPS}

Temprano en la vida del arbitraje de inversión, los tribunales tuvieron que dedicar su atención al tratamiento del acceso a la información y la participación de terceros en el arbitraje. Esta problemática se manifestó, especialmente, en los casos arbitrales sustanciados bajo el Capítulo XI del TLCAN. En Metalclad $c$. México el Tribunal estableció que ni bajo el TLCAN ni bajo los Mecanismos Complementarios del CIADI existía regla alguna que limitara a las Partes la libertad de hacer públicos documentos que hayan sido objeto del juicio arbitral. ${ }^{8}$ En Loewen $c$. EE.UU. el Tribunal sostuvo que no era deseable una regla general sobre confidencialidad en el arbitraje con parte estatal, en la medida que restringiría el acceso público a información que refiere a cuestiones de gobierno y de interés público.?

Como consecuencia de todos estos antecedentes, en el año 2001, la "Comisión de Libre Comercio" del TLCAN dispuso:

1. Nada de lo dispuesto en el TLCAN impone una obligación general de confidencialidad para las partes contendientes en un arbitraje al amparo del capítulo XI, y, sujeto a la aplicación del artículo 1137(4), nada de lo dispuesto en el TLCAN impide a las Partes otorgar acceso al público a documentos presentados ante un tribunal constituido conforme al capitulo XI o expedidos por él.

2. En aplicación de lo anterior:

(a) De conformidad con el artículo 1120(2), las Partes del TLCAN acuerdan que nada de lo dispuesto en las reglas de arbitraje pertinentes impone una obligación general de confidencialidad o impide a las Partes otorgar acceso al público a documentos presentados ante un tribunal constituido conforme al Capítulo XI, o expedidos por él,

8. Metalclad Corporation c. Estados Unidos Mexicanos. Caso CIADI ARB(AF)97/! (Lauterpacht,Civiletti. Siqueiros). Decisión del 30 de Agosto de 2000, 13.

9. The Loewen Group Inc and Raymond L. Loewen c. Estados Unidos de América. Caso CIAD1 ARB(AF) 98/3. (Mason. Fortier,Afirka). Decisión del 5 de enero de 2001. \$26. 
salvo las excepciones específicas y limitadas previstas expresamente en esas reglas. $(. . .)^{10}$

En Methanex c. EE.UL.,"1 el Tribunal tuvo que referirse a cuestiones de transparencia diferentes a las referidas en los casos Metalclad y Loetwen; más precisamente, a la participación de amicus curiae en el procedimiento arbitral. En términos generales, las determinaciones del Tribunal en Methanex, aplicando el Artículo 15 de las Reglas de Arbitraje CNUDMI, son similares a las que posteriormente adoptaría un Tribunal en otro caso bajo el Capítulo XI del TLCAN: UPS c. Canadá. ${ }^{12}$

Ambos Tribunales ${ }^{13}$ determinaron que tenían jurisdicción para resolver la participación de amicus curine bajo los amplios poderes a ellos otorgados bajo la Regla 15 de las Reglas de Arbitraje de la CNUDMI.

\subsubsection{Aguas del Tunari c. Bolivia y AASA y APSF (Suez) c. República Argentina}

Antes de la Reforma a las Reglas de Arbitraje ocurrida en el año 2006, inclusive bajo las Reglas de Arbitraje del CIADI -que parecen ser las que originalmente eran las más ajustadas para regular la materia específica del arbitraje de inversión y, en tal sentido permitir alguna forma de participación y transparencia-, establecían que las audiencias se desarrollarían a puertas cerradas y que sólo podían participar de ellas las partes, sus agentes, asesores, abogados, testigos y expertos. En función de esta norma el Tribunal en Aguas del Tunari c. Bolivia decidió, ante un pedido realizado por un grtupo de interés para participar en el

10. Nota Interpretativa de la Comision de Libre Comercio del TLCAN. http:/www.economia gob.mx $/$ es/cconomia/p_solucion_controversias_inversionista.

11. Methanex c. Estados Unidos de América. Caso CNUDMI. (Vecder,Rowley:Reisman). Decisión sobre Jurisdicción y Aưmisibilidad del 7 de agosto de 2002.

12. United Parcels Service of America, Ine (UPS) c. Canadá, Caso UNCITRAL. (Kieith.Forticr,Cass). Decisión sobre la Participación de Amici Curiae. 17 de octubre de 2001.

13. Por conveniencia, trato aqui ambas decisiones en conjunto, sin desconocer que ambas tienen sus propias caracteristicas particulares. 
procedimiento arbitral, que no estaba en su poder autorizar la participación si no existía el consentimiento de ambas partes.

El Tribunal en Aguas Argentinas Sociedad Anónima y Suez (AASA), Aguas de Barcelona SA, Vivendi Universal SA y AWG c. Reptiblica Argentinal4 aceptó la participación de amicus curiae aunque negó la participación de estos en la audiencia y el acceso a documentos existentes en el expediente. ${ }^{15} \mathrm{El}$ Tribunal reguló en detalle la participación de los amici y los condicionó -al igual que lo habian hecho los tribunales en Methanex y UPS- a que éstos acerquen al procedimiento elementos de hecho o de derecho que ayuden al Tribunal a resolver el caso. Asimismo, intentó a través del procedimiento regulado no hacer más gravoso el ejercicio de la defensa por las Partes en el arbitraje.

El mismo Tribunal entendió en el caso Aguas Provinciales de Santa Fe (APSF) y Suez, Aguas de Barcelona SA, e Interaguas Servicios del Agua c. República Argentina, ${ }^{16}$ y negó allí la participación de los amici, justamente por no cumplir con las condiciones a la que sujetó la participación de éstos en el caso referido en el párrafo anterior.

\subsubsection{La Reforma de las Reglas de Arbitraje del CIADI de 2006 BiWater Gauff}

En el año 2006, el Consejo Administrativo del CIADI realizó una reforma integral de las Reglas de Arbitraje del CIADI. ${ }^{17} \mathrm{La}$ Secretaría del CIADI, el grupo de trabajo elegido para llevar ade-

14. Aguas Argentinas Sociedad Anónima y Suez (AASA), Aguas de Barceiona SA, Vivendi Universal SA c. República Argentina. Caso CIADI ARB/03/19, y AWG c. República Argentina (Caso CNUDM1). (Salacuse, Kauffman-Kohter,Nikken). Decisión sobre la Participación de Amicus Curiae.

15. La República Argentina apoyó la participación de los amici en las Audiencias, pero siendo que la Parte Demandante negó tal participación el Tribunal no hizo lugar al pedido de las ONGs. En tal sentido ver Aurelia Antonietti, THE 2006 AMENDMENTS OF THE ICSID RULESS AND REGULATIONS AND THE

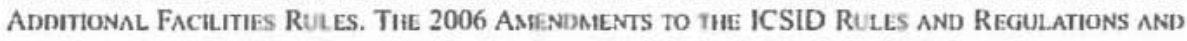
TiE ADIITIONal Faci ity Rule. ICSID Review - FIL, Vol. 21, No. 2, Fall 2006, р. 6.

16. Aguas Provinciales de Santa Fe y Suez (APSF), Aguas de Barcelona SA, e Interaguas Servicios del Agua c. Repüblica Argentina. Caso ClADI ARB/03/17. (Salacuse, Kauffman-Kohler. Nikken). Decisión sobre la Participación de Amicus Curiue. 
Ignacio Torierola

lante las reformas y las demás personas que fueron consultadas en el proyecto entendieron que la temática de la "transparencia y participación de terceros" estaban entre las cuestiones que demandaban una revisión de las Reglas de Arbitraje, para adaptarlas a las nuevas necesidades que surgían del mayor número de casos y de la circunstancia que el consentimiento arbitral se había dado en un tratado. ${ }^{18}$

En tal sentido, tres nuevas reglas, que intentaban reflejar el estado de la práctica arbitral de inversión, fueron aprobadas. La Regla 32 sobre "apertura de las audiencias", la Regla 37(2) sobre participación de amicus curiae y el artículo 48 sobre publicación del laudo arbitral. La Regla 32(2) dispone, respecto de las audiencias:

(2) Salvo objeción de alguna de las partes, el Tribunal, tras consultar con el Secretario General, podrá permitir, sujeto a los arreglos logísticos pertinentes, que otras personas, además de las partes, sus apoderados, consejeros y abogados, testigos y peritos durante su testimonio, y funcionarios del Tribunal, asistan a la totalidad o parte de las audiencias, o las observen. En dichos casos el Tribunal deberá establecer procedimientos para la protección de información privilegiada o protegida.

17. Aurclia Antonicti. The 2006 Amendments of the ICSID Rules and Regulations and tiIr: AdDitional Facilities Rules. Op. Cit. p. 2. Antonietti sostiene que "con el incremento de casos frente al CIADI y el crecimiento de su jurisprudencia, se hizo palpable la necesidad de introducir modificaciones a las Reglas de Arbitraje del CIADI para que estas pudieran satisfacer las necesidades de los usuarios del Centro, particularmente en relación a los casos que se iniciaban bajo la invocación de los tratados bilaterales o acuerdos multilaterales de inversión. En tal sentido, se habian presentado quejas respecto a la faha de transparencia, y en tal sentido- fue expresada la necesidad de codificar las prácticas habituales de los tribunales CIADI. Se propusieron cambios que tenian por objetivo introducir esos cambios y que refiejaran la práctica de los tribunales. A los fines de introducir csos cambios, ta Secretaria del CIADI se benefició de las consultas que hizo a miembros del Comité Consultivo del Banco, a asociaciones profesionales, grupos de interés, experto en arbitraje internacional y a instituciones a través del mundo." Ver en tal sentido, Antonicui, Op. Cit. p. 3.

18. Aurelia Antonietti, Op. Cit, página 3. Como es ampliamente reconocido, el ClADI se creó con la idea de su aplicación a controversias de inversión en las cuales el consentimiento habia sido dado a través de una cláusula contractual. Esta circunstancia hace que los hechos que se discuten en un arbitraje CIADI actual puedan potencialmente tener un impacto mucho más importante sobre el interés público que una controversia puntual por una cuestión comercial -con una parte cstatal-conicnida en un contrato. 
La Regla 37(2) dispone respecto a la participación de amicus curiae en el procedimiento arbitral:

(2) Después de consultar a ambas partes, el Tribunal puede permitir a una persona o entidad que no sea parte en la diferencia (en esta regla "parte no contendiente") que efectúe una presentación escrita ante el Tribunal, relativa a cuestiones dentro del ámbito de la diferencia.

Al determinar si permite dicha presentación, el Tribunal deberá considerar, entre otras cosas, en qué medida:

(a) la presentación de la parte no contendiente ayudaría al Tribunal en la determinación de las cuestiones de hecho o de derecho relacionadas con el procedimiento al aportar una perspectiva, un conocimiento o una visión particulares distintos a aquéllos de las partes en la diferencia;

(b) la presentación de la parte no contendiente se referiría a una cuestión dentro del ámbito de la diferencia;

(c) la parte no contendiente tiene un interés significativo en el procedimiento.

El Tribunal deberá asegurarse de que la presentación de la parte no contendiente no perturbe el procedimiento, o genere una carga indebida, o perjudique injustamente a cualquiera de las partes, y que ambas partes tengan la oportunidad de someter observaciones sobre la presentación de la parte no contendiente.

Finalmente, la nueva Regla 48 dispone:

(4) El Centro no publicará el laudo sin el consentimiento de las partes. Sin embargo, el Centro deberá incluir prontamente en sus publicaciones extractos del razonamiento jurídico del Tribunal.

El Tribunal en Biwuter Gauff fue el primero en aplicar las nuevas normas del CIADI en materia de transparencia y acceso a la información. Dos cuestiones distintas se presentaron para la resolución del Tribunal: (a) la publicación de documentos pre- 
sentados por las partes en el arbitraje (y el acceso a ellos por terceras partes) y, (b) la participación de amicus curine en el arbitraje. Debe notarse, en tal sentido, que las nuevas reglas del CIADI no contienen normas sobre publicación de documentos y que estas cuestiones han quedado en gran parte limitadas a las disposiciones que en tal sentido pueda contener el acuerdo arbitral que da lugar a la controversia.

El Tribunal permitió la participación de los amici en cuanto consideró que existía un interés público en la controversia y que la participación de los amici favorecería la aceptación del resultado del arbitraje y ayudaría al Tribunal a cumplir con sus obligaciones. ${ }^{19}$ Por otra parte, el Tribunal no permitió la participación de los amici en la audiencia ${ }^{20} \mathrm{O}$ el acceso de estos a los documentos del proceso arbitral.21

\subsubsection{El Artículo 34(5) de las Reglas de Arbitraje CNUDMI 2010}

El nuevo artículo 34(5) de las Reglas de Arbitraje de la CNUDMI dispone:

34(5) Podrá hacerse público el laudo con el consentimiento de las partes o cuando una parte tenga la obligación jurídica de darlo a conocer para proteger o ejercer un derecho, y en la medida en que así sea, o con motivo de un procedimiento legal ante un tribunal u otra autoridad competente.

La Regla 32(5) anterior establecía, respecto a la publicidad del laudo, que este sólo podía hacerse público con el consentimiento de las partes. La nueva Regla 34(5), siguiendo el modelo adoptado por la Corte Internacional de Arbitraje de Londres (LCIA), introduce la posibilidad de que el laudo sea hecho públi-

19. Biwater Gauff c. República Unida de Tanzania. Caso CIADI ARB 05/22 (Hanotiau, Born. Lamedau). Orden Procesal No. 5 del 2 de febrero de 2007, $\$ 47$ y siguientes.

20. Biwater Gauff, Orden Procesal No. 5, \$ 72.

21. Biwaler Gauff, Orden Procesal No. 5, $\$ 68$. 
co, además, cuando este refleje una obligación legal, situación esta última que alcanza a la mayoría de los Estados modernos.

Precisamente, la nueva norma contenida en el Artículo 34(5) refleja, justamente, la obligación legal que algunas partes en el arbitraje pueden tener como consecuencia de obligaciones legales independientes del arbitraje. En el caso de los Estados, como se ha visto anteriormente en la sección (b) de este Capítulo, éstos pueden tener obligaciones legales de acceso a la información y de publicidad de los actos de gobierno.

Finalmente, el sistema de cortes nacionales generalmente se desarrolla en forma totalmente pública y, como resultado de las acciones que las partes pueden ejercer judicialmente en apoyo del arbitraje, es posible que una decisión arbitral termine siendo sometida a uno o más sistemas de justicia nacional, transformándose así en una información que existe en el dominio público.

\subsection{Hacia la Conformación de un Cuerpo Jurisprudencial}

Las decisiones de los tribunales arbitrales han creado progresivamente un cuerpo jurisprudencial que debe ser tenido en consideración porque derivan conclusiones de la realidad económica y conforme a las necesidades del comercio internacional respecto a las cuales el arbitraje internacional debe responder ... (Dow Chemical France v. Isover Saint Gobain, Caso CCI No. 4131).22

Desde hace un tiempo he venido expresando la idea que todas las decisiones arbitrales deberían tener cierta forma de

publicidad. Cuando refiero a las decisiones arbitrales no me

22. Caso CCI No. 4131, Dow Chemical France v. Isover Saint Gobain, Interim Award del 23 de septiembre de 1982, IX Y.B. Com. Arb. 131, 136 (1984): CA Paris, Oct.21, 1983, Isover-Saint-Gobain v. Dow Chemical France, 1984 Rev. Arb. 98 (Anotado por A. Chapelie). Mourre, Alexis. Precedent and Confidentiality in international. Commercial. Aruitration. Tile Case for the Publication of ARbitral Awards, en Precedent in Intemational Arbitration. IAI Serics, Yas Banitafemi (editor), p. 45. 
limito a las decisiones en materia de arbitraje de inversión sino que incluyo también la publicidad de cualquier tipo de decisión arbitral, inclusive las que tienen un carácter estrictamente comercial y que carecen de la participación de parte estatal. La razón es simple: la publicidad de las decisiones arbitrales crea un cuerpo jurisprudencial ${ }^{23}$ que ayuda a la calidad y consistencia de las decisiones arbitrales y a la confianza que generan en sus usuarios, no solamente aquellos habituados a recurrir a este mecanismo sino a un público más amplio que está encontrando el atractivo del arbitraje pero que desconfía del "secretismo" que rodea a la práctica arbitral y que, ciertas veces, lo transforma en algo casi esotérico. Lo ocurrido con el arbitraje de inversión en la última década prueba que la publicidad ayuda a hacer más previsible el sistema, y reduce las ineficiencias e imprevistos que las partes pueden encontrar en el futuro. Además, eleva los estándares profesionales requeridos de los árbitros en sus decisiones.

Sin saberlo, esta idea que imaginé revolucionaria, ya había sido expresada por otros juristas que también habían pensado en la necesidad de dar publicidad a las decisiones arbitrales, a los fines de formar un cuerpo jurisprudencial, elevar la calidad de las decisiones y ayudar-de esa manera- a la previsibilidad y confianza de las partes (y el público en general) en relación al arbitraje como medio de solución de controversias. ${ }^{2+}$ En tal sentido, dos obras de obligatoria lectura son MOURRE 25 y LEW26 en "The Case for the Publication of Arbitral Awards". Lew expresa claramente la conveniencia de esta idea en los siguientes términos:

23. Cuando refiero a cuerpo jurisprudencial no necesariamente intento afirmar que estas decisiones tengan caraicter de precedentes, sino simplemente de guia autorizada a los fines de crear un sistema que provea la mejor calidad posible de decisiones.

24. Esto debe leerse en oposiciōn al ritmo de las publicaciones que las "instituciones arbitrales" dan a la publicidad de sus decisiones.

25. Moutrc, Alexis. Precedent and Confidentiality in Intehnational. Commercial. Aribitration. The Case fok the Publication of arbitral awards. in Precedent in International Arbitration. Iai Series, Yas Banitafemi, Editor, 2008.

26. Julian D.M. Lew, The Case for the Publication of Arbitration Awards, in thl. ARt of arbitration - Essay on International arbitration - Liber amicorum Pieter Sanders 12 Sertemiber 1912 -. J.C. Schultsz y A.J. van den Berg (editores) Kluwer, 1982, p. 223. 
la publicación de las decisiones arbitrales identificará las verdaderas ventajas del arbitraje: especialistas y expertos en el arbitraje operando a nivel internacional. El desarrollo de un cuerpo jurisprudencial dará al arbitraje una mayor certeza de la que actualmente existe con respecto a la dirección que los árbitros seguirán y facilitará la aceptación del derecho comercial internacional y la aceptación de la lex mercatoria. Ello casi seguramente resolverá problemas que recurrentemente se le presentan a los árbitros e influenciará la actitud negociadora y las decisiones de los hombres de negocios. Más importante aún, la publicación sistemática de laudos arbitrales demostrará que el arbitraje no solo es una alternativa viable a las cortes internacionales como medio de solución de controversias sino que además demostrará concluyentemente que el arbitraje es más apropiado como medio de solución de controversias que surgen del comercio internacional. ${ }^{27}$

MOURRE identifica ciertos aspectos que entiendo es necesario considerar. Sostiene que es necesario un sistema que permita la publicación del mayor número de casos y con las menores omisiones posibles. Sólo un número suficientemente amplio de casos puede dar lugar a la conformación de un cuerpo jurisprudencial ${ }^{28} \mathrm{El}$ acceso a otras decisiones arbitrales mejoraría la calidad de nuevas decisiones arbitrales y permitiría una identificación más clara de los principios jurídicos que cumplen un rol central en el proceso arbitral.29 Finalmente, sostiene la necesidad

27. (Traducción del autor) "[t]he publication of arbitrations awards would ... identify the real advantages of arbitration: specialist and expert arbitrators operating on the international level. The development of an arbitral case law would give to arbitration a greater certainty than that presently existing, with respect to the probable attitude of the arbitrators, and would facilitate the commercial world's knowledge and acceptance of the lex mercatoria. This would almost certainly obviate many recurring problems presented to arbitrators and would influence the negotiating attitudes and commercial decisions of businessmen. Above all, the systematic publication of arbitration awards would show that not only is arbitration an altemative to national courts as a system of dispute settlement, but it would prove conclusively that arbitration is the most appropriate forum in which to resolve disputes arising out of intemational commerce. Julian D.M. Lew, Thie CASE for the Publication of Arbitration AWARDS, cn THE: ART OF ARIMTRATION - ESSAY ON INTERNATIONAL ARBITRATION - LIBER AMIICORLM Pieter Sanders 12 Septentber 1912 - 1982, J.C. Schulisz y A.J. van den Berg (editores) Kluwer, 1982. citado por Mourre, A. Op. Cit. p. 223.

28. Mourre, Op. Cit. p. 48. Para apoyar esta afirmación Mourte cita a Christian Larroumet, A propos DE l. Jurisprudence Artitrale, Gaz. Pal. Dec.14, 2006. 
de conocer el nombre de los árbitros, por el prestigio asociado a estos, lo cual daría mayor o menor relevancia a la decisión (así como mayor previsibilidad y consistencia al sistema arbitral). ${ }^{31}$

No puedo sino coincidir con todas las apreciaciones hechas en los párrafos anteriores.

\section{Conclusiones}

Cada tribunal actuando en un caso que incluye una parte Estatal, especialmente si la jurisprudencia de ese tribunal arbitral proviene de un tratado bilateral de inversiones, es - en opinión de este autor - una corte internacional. Nadie ha indicado que características puede tener la jurisdicción de la Corte Internacional de Justicia que puedan estar ausentes en un tribunal de inversión. En ambos casos la jurisdicción proviene de un acuerdo internacional, las medidas que dan lugar a la jurisdicción proviene de una medida de éste tomada en ejercicio de actividad iure imperii. En muchos casos, inclusive, los árbitros o miembros de los tribunales son los mismos y sus nombres provienen (especialmente en el caso del CIADI) de una lista nombrada por Estados soberanos.

Con la debida protección de información confidencial y de alguna otra información que pueda ser excesivamente perjudicial para una o ambas partes, los Tribunales de Inversión tienen la obligación de permitir un suficiente acceso a la información que se debe manifestar, como mínimo en las siguientes obligaciones: registro de la existencia de la disputa, participación de amigos de la Corte cuando estos puedan ofrecer una perspectiva distinta a la presentada por las Partes y auxiliar al tribunal. Finalmente, también se debe proveer a los medios para la publicación de la decisión. Ello asegura que los jueces (árbitros) ejer-

29. Mourre, Op. Cil. p. 49.

30. Mourre, Op. Cit. p. 58. 
citen sus funciones con la responsabilidad que requiere las altas funciones que les han sido encomendadas. 